\title{
ON IMPLICIT MODELING FOR FITTING PURPOSES
}

\author{
Ralf Plänkers \\ Pascal Fua* \\ \{Ralf.Plaenkers,Pascal.Fua\}@epfl.ch \\ Computer Graphics Lab (LIG) \\ Swiss Federal Institute of Technology (EPFL) \\ CH 1015 Lausanne
}

Keywords: Body fitting, body modeling, implicit surface, metaball

\begin{abstract}
Tracking and modeling people from video sequences has become an increasingly important research topic, with applications including animation, surveillance and sports medicine. In this paper, we propose a model based 3-D approach to recovering both body shape and motion. It takes advantage of a sophisticated animation model to achieve both robustness and realism. Stereo sequences of people in motion serve as input to our system. From these, we extract a 2.5-D description of the scene and, optionally, silhouette edges. We propose an integrated framework to fit the model and to track the person's motion. Constraints for 3-D points and silhouette edges are presented in detail. We recover not only the motion but also a full animation model closely resembling the subject.
\end{abstract}

\section{Introduction}

Tracking and modeling people from video sequences has become an increasingly important research topic, with applications including animation, surveillance and sports medicine. In this paper, we propose a 3-D approach to recovering both body shape and motion. We obtain stereo- and silhouette-data from synchronized cameras and we fit to it a sophisticated body model. We use it to eliminate erroneous data, to

*'The work reported here was funded in part by the Swiss National Science Foundation. 
A detailed description of the human body in the form of an animated layered model is at the root of our work. It provides a priori information about the shape, and the allowable motions of the human body. This is essential for interpreting noisy data and solving the resulting ambiguities. The model we use is made of volumetric primitives attached to an articulated skeleton (Thalmann et al., 1996). This implicit surface formulation has several advantages, among them a lower number of parameters and a 3-D distance measure that is differentiable and fast to compute.

As input to our system we use image sequences of people in motion. Multiple synchronized and calibrated cameras are used to extract stereo information. Because cameras are relatively cheap and disparity maps such as the ones we use can be acquired at frame rate on ordinary computers (Konolige, 1997), this is not a major limitation for many applications. Also, stereo works well both on textured clothes and on bare skin. Silhouette edges can be included when available. Stereo and silhouettes are complementary sources of information: Stereo works well where the surface faces the camera but fails where the surface slants away. Silhouettes, on the other hand, provide information exactly there, at the occluding contour.

We have developed an extensible least squares framework that we use to fit the body model to the different types of input data, with minimal human intervention. To initialize the process, the user simply clicks on the approximate location of a few key-points in one image pair. The recovered shape and motion parameters can then be used to reconstruct the original motion, to display it from a different viewpoint or to make other animation models mimic the subject's actions.

Recently, techniques have been proposed to track human motions from video sequences. They are fairly effective but use very simplified models of the human body, such as ellipsoids or cylinders, that do not precisely model the human shape. The recovered motion can indeed be applied to other models. However, a model of the filmed person that would be sufficient for a truly realistic animation is not obtained. The interested reader is referred to the recent surveys in (Gavrila, 1999; Moeslund and Granum, 2001) for further references.

Automatic (Hilton et al., 1999) and semi-automatic (Lee et al., 2000) systems for 3-D model acquisition from orthogonal photographs have been developed recently. They work fairly well for the intended applications like populating virtual worlds. However, most of the realism is due to texture mapping while the geometry is only a crude approximation.

While laser scanning technology provides a fairly good surface description of a static object, using video sequences allows us in addition 
to measure and track the person in motion and, thus, to recover the positions of the articulations inside the skin surface.

\section{Body Model}

The animated body model we use is made of volumetric primitives called metaballs attached to an articulated skeleton. Each one generates a potential field and the skin is taken to be an isosurface of the combined potential (Thalmann et al., 1996).

\subsection{State Vector}

Our goal is to use video-sequences to estimate our model's shape and derive its position in each frame. Let us therefore assume that we are given $N$ consecutive video frames and introduce position parameters for each frame.

Let $B$ be the number of body parts in our model. We assign to each body part a variable length and width coefficient. These dimensions change from person to person but we take them to be constant within a particular sequence. This constraint could be relaxed, for example to model muscular contraction.

The model's shape and position are then described by the combined state vector

$$
\Theta=\left\{\Theta^{w}, \Theta^{l}, \Theta^{r}, \Theta^{g}\right\}
$$

where we have broken $\Theta$ into four subvectors which control the following model components:

- Shape.

- $\Theta^{w}=\left\{\theta_{b}^{w} \mid b=1 . . B\right\}$, the width of body parts.

- $\Theta^{l}=\left\{\theta_{b}^{l} \mid b=1 . . B\right\}$, the length of body parts.

- Motion.

- $\Theta^{r}=\left\{\theta_{i, f}^{r} \mid j=1 . . J, f=1 . . N\right\}$, the rotational degree of freedom of joint $j$ of the articulated skeleton for all frames $f$

- $\Theta^{g}=\left\{\theta_{f}^{g} \mid f=1 . . N\right\}$, the six parameters of global position and orientation of the model in the world frame for all frames $f$

The size and position of the metaballs is relative to the segment they are attached to. A length parameter not only specifies the length of 
a skeleton segment but also the shape of the attached metaballs in the direction of the segment. Width parameters only influence the metaballs' shape in the other directions.

Motion paramaters $\Theta^{r}$ are represented in terms of Euler angles. We can constrain joint motions to anatomically valid ranges by defining an allowable interval for each of the degrees of freedom. Other methods for describing rotations, such as quaternions or exponential maps, can be used as well.

\subsection{Metaballs}

Metaballs are defined by a set of points that are the sources of a potential field. Each source is defined by a field function $F_{i}(x, y, z)$ that maps $\mathbb{R}^{3}$ to $\mathbb{R}$. At a given point $\mathbf{x}(x, y, z)$ of the Euclidean space, the fields of all sources are computed and added together, leading to the global field function

$$
F(x, y, z)=\sum_{i=1}^{n} F_{i}(x, y, z)
$$

Choosing a threshold value $T$ then allows us to define the implicit surface

$$
\mathbf{S}=\left\{(x, y, z) \in \mathbb{R}^{3} \mid F(x, y, z)=T\right\}
$$

We take the field function $F_{i}$ to be

$$
F_{i}(x, y, z)=f_{i}\left(d_{i}(x, y, z)\right)
$$

where $d_{i}$ is a distance function that maps $\mathbb{R}^{3}$ to $\mathbb{R}^{+}$, and $f_{i}$ is a potential function which maps $\mathbb{R}^{+}$to $\mathbb{R}$ (Blanc and Schlick, 1995). The function $d_{i}$ characterizes the distance between a given point $\mathbf{x}(x, y, z)$ and the source point of a metaball. The most obvious choice is the Euclidean distance, but several other functions have been proposed in the literature.

In this work, we take $d_{i}$ to be an algebraic ellipsoidal distance:

$$
d_{i}(x, y, z)=\left(\frac{x-\theta_{i}^{w} c_{x, i}}{\theta_{i}^{w} l_{x, i}}\right)^{2}+\left(\frac{y-\theta_{i}^{w} c_{y, i}}{\theta_{i}^{w} l_{y, i}}\right)^{2}+\left(\frac{z-\theta_{i}^{l} c_{z, i}}{\theta_{i}^{l} l_{z, i}}\right)^{2},
$$

where $L_{i}=\left(l_{x, i}, l_{y, i}, l_{z, i}\right)$ are the radii of ellipsoid $i$, i.e. half the axis length along the principal directions and $C_{i}=\left(c_{x, i}, c_{y, i}, c_{z, i}\right)$ is the primitive's center. Coefficients $\theta_{i}^{l}$ and $\theta_{i}^{w}$ from the state vector $\Theta$ control relative length and width of a metaball. They are shared among groups of metaballs according to segment assignment. For simplicity's sake, in 
the remainder of the paper, we will omit the $i$ index for specific metaballs wherever the context is unambiguous.

We use ellipsoidal primitives because they are simple and, at the same time, allow accurate modeling of human limbs with relatively few primitives because metaballs result in a smooth surface, thus keeping the number of parameters low. Using algebraic distances for fitting purposes can result in overfitting in the high-curvature regions in some cases (Sullivan et al., 1994). For our specific application, however, the ellipses only have limited degrees of freedom and are rigidly attached to a skeleton structure. Their shape is controlled by higher level width and length parameters, and, thus, such problems do not occur.

To permit an effective fit of our implicit surface model to the data we use an exponential field function:

$$
f_{i}=\left(\frac{1}{e^{d_{i}}}\right)^{2}=\exp \left(-2 d_{i}\right) .
$$

Function $f_{i}$ is differentiable over the whole domain and it has a long range effect because it approaches zero slowly. In the context of model fitting these two properties are very important as will be discussed in Section 2.

\section{Fitting the Models to Image Data}

From a fitting point of view, the body model embodies a rough knowledge about the shape of the body and can be used to constrain the search space. Our goal is to derive its degrees of freedom so that it conforms as faithfully as possible to the image data.

Here we use motion sequences such as the ones shown in Figure 2. Silhouette information can be added when available, as shown in 1. Thus, the expected output of our system is a state vector that describes the shape of the metaballs and a set of joint angles corresponding to their positions in each frame.

\subsection{Least Squares Framework}

In standard least-squares fashion, we use the image data to write nobs observation equations of the form

$$
y_{i}(S)=o b s_{i}-\epsilon_{i}, 1 \leq i \leq n o b s
$$

where $S$ is the state vector of Eq. 1 that defines the shape and position of the limb and $\epsilon_{i}$ is the deviation from the model. We will then minimize

$$
v^{T} P v \Rightarrow \operatorname{Min},
$$


where $v=\left[\epsilon_{1}, \ldots, \epsilon_{n o b s}\right]$ is the vector of residuals and $P$ is a weight matrix associated with the observations. $P$ is usually introduced as diagonal.

Our system must be able to deal with observations coming from different sources that may not be commensurate with each other. Formally we can rewrite the observation equations of Equation 7 as

$$
y_{i}^{\text {type }}(S)=o b s_{i}^{\text {type }}-\epsilon_{i}, 1 \leq i \leq n o b s,
$$

with weight $p_{i}^{\text {type }}$, where type is one of the possible types of observations we use. In this paper, type can be object space coordinates or silhouette rays. However, other information cues can easily be integrated.

The individual weights of the different types of observations have to be homogenized prior to estimation according to:

$$
\frac{p_{i}^{k}}{p_{j}^{l}}=\frac{\left(\sigma_{j}^{l}\right)^{2}}{\left(\sigma_{i}^{k}\right)^{2}}
$$

where $\sigma_{j}^{l}, \sigma_{i}^{k}$ are the a priori standard deviations of the observations $o b s_{i}, o b s_{j}$ of type $k, l$.

Least-squares estimation means finding the joint minimum

$$
\sum_{\text {type }=1}^{n t} v^{\text {type }} P_{t y p e} v^{\text {type }} \Rightarrow M i n
$$

where $n t$ is the number of observation types. It yields the well-known normal equations which need to be solved using standard techniques.

Since our overall problem is non-linear, the results are obtained through an iteration process. We use an implementation of the LevenbergMarquardt algorithm (Press et al., 1986) that can handle the large number of parameters and observations we must deal with.

\subsection{3-D Observations}

Each 3-D point reconstructed using stereo or similar techniques is introduced as one observation into the system. The point has to lie on the surface of the model in order to be explained by the model. It's objective function, i.e. the error in the model and thus the distance between point and model, has to be minimized. We use the value of the implicit field function as algebraic distance and minimize it instead of a geometric distance. Please refer to (Plänkers and Fua, 2001) for a description of how to compute the Jakobians. 


\section{3. $\quad$ From Silhouette Data to Observations}

Contrary to $3-\mathrm{D}$ edges, silhouette edges are typically $2-\mathrm{D}$ features since they depend on the viewpoint and cannot be matched across images. However, they constrain the surface tangent. Each point of the silhouette edge defines a line, the camera ray, that goes through the optical center of the camera and is tangent to the surface at its point of contact with the surface. The points of a silhouette edge therefore define a ruled surface that is tangent to the surface to be modeled.

3-D position of silhouette edges The main difficulty is to find the metaball surface point $X$ where the constraint applies. In practice, we take this point to be the point on the camera ray which minimizes the implicit formulation of the model, Eq. 6. This point depends on the position of the model and we have to adjust the Jakobian of the constraint in order to account for the model dynamics.
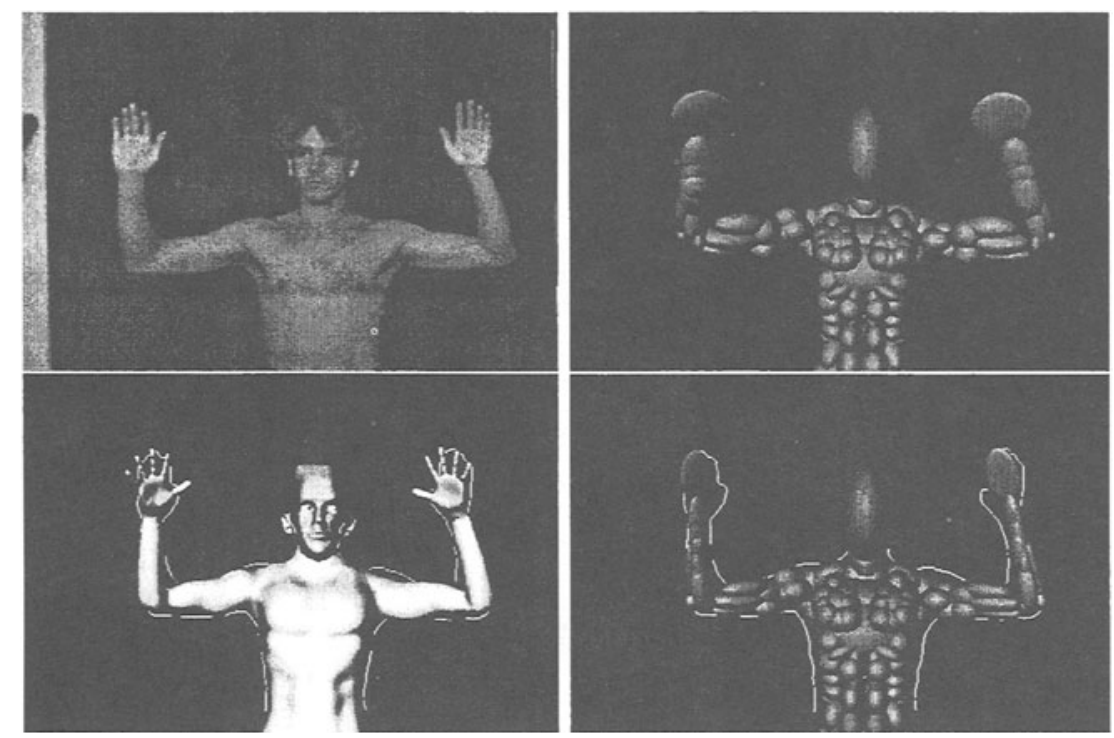

Figure 1. The importance of silhouette information for shape modeling. The original image is shown in the upper left. In the upper right no silhouette constraints were used and the fitting puts the model is too far away from the cloud. This is compensated by enlarging the primitives. The silhouettes provide stricter constraints for the model. The lower row shows the result of the fitting with and without skin rendered.

The importance of using silhouette information is demonstrated by Figure 1. Here, we allowed for changes in the model's posture and the shape parameters of the arms. In the upper row of Figure 1 only the 
3-D information is used. The fitting tends to move the model further away from the cloud and to compensate by inflating the arms to keep contact with the point cloud. The noisy stereo data is too ambiguous to sufficiently constrain the model. The silhouettes are needed to constrain it, as shown in the lower row of Figure 1 where we fitted to both stereo and silhouette information.

\section{Combining Articulated Structure and Implicit Surfaces}

\subsection{The Modular Approach}

We combine metaballs and articulated skeleton by defining the observation $\mathbf{x}=[x, y, z]^{T}$ as being a function of the skeleton structure. This way, the derivatives wrt. to the parameters of the articulated structure and those local to the metaballs can be separated by simple application of the chain rule. Following is a description of deriving derivatives for the common Euler-Angle formulation of rotational joints. Here, a joint can only have a rotation around a single fixed axis. More complicated joints can be modeled by several single-DoF joints sharing the same position but having different orientations.

Euler-Angles. For some distance function $d\left(\mathbf{x}, \theta^{r}\right)$ with $\mathbf{x}=[x, y, z]^{T}$ being the observation in local coordinates of joint $j$ and $\theta^{r}=\left\{\theta_{j}^{r}\right\}, j=$ 1 ..J the rotational joint parameters of the object we can write the jacobian entries wrt. to each DoF $\theta_{j}^{r}$ as

$$
\frac{\partial d}{\partial \theta_{j}^{r}}=\frac{\partial d}{\partial \mathbf{x}} \cdot \frac{\partial \mathbf{x}}{\partial \theta_{j}^{r}}
$$

The first term $\frac{\partial d}{\partial \mathbf{x}}$ only depends on the chosen distance function. The second term $\frac{\partial \mathbf{x}}{\partial \theta_{j}^{r}}$ expresses the change induced by the rotation at joint $j$. Decomposing a rotational joint into a directional axis a and an angle value $\theta_{j}^{r}$, i.e. the amount of rotation around this axis, we can rewrite the second term as

$$
\frac{\partial \mathbf{x}}{\partial \theta_{j}^{r}}=\mathbf{a}_{j} \times \overrightarrow{\mathbf{O}_{j} \mathbf{O}_{E}}\left(\theta_{j}^{r}\right)
$$

with $\overrightarrow{\mathbf{O}_{j} \mathbf{O}_{E}}$ being the vector from the joint $j$ 's reference frame to the end-effector under the current rotation $\theta_{j}^{r}$. All vectors are expressed in global coordinate frames. In the case of an observation the vector 
needs to be inverted to yield $\overrightarrow{\mathbf{O}_{o b s} \mathbf{O}_{j}}$. For proof we refer the interested reader to (Baerlocher and Boulic, 1999). For Euler-Angles this Eq. 13 can directly be used by repeating it for each axis that allows a rotation (omega, phi, kappa).

Metaball Parameters. Local metaball parameters, like size or position which are independ of the skeleton posture are derived as follows. For some distance function $d\left(\mathbf{x}, \theta^{m}\right)$ with $\mathbf{x}=[x, y, z]^{T}$ being the observation and $\theta^{m}=\left\{\theta_{i}^{m}\right\}, i=1 . . I$ the local metaball parameters of the object we can write the jacobian entries wrt. to each DoF $\theta_{i}^{m}$ as

$$
\frac{\partial d}{\partial \theta_{i}^{m}}
$$

This means the skeleton structure remains constant and $\mathbf{x}$ can simply be used as already transformed into joint local coordinates, according to the current skeleton configuration. The derivation becomes straightforward.

\subsection{Discussion}

Separability of the single modules is a clear advantage when it comes to implementation of the method described above. Code reuse and simplicity of functions greatly increase reliability and ease of testing. However, the use of this technique becomes more problematic when combined influences need to be addressed. How do we formulate complex derivatives that describe the interplay of parameters of different types? This problem arises when integrating 2-D silhouette constraints in our 3-D fitting framework. The point on the 3-D silhouette ray that is closest to the model, i.e. the point of intersection with the real-world object, moves along the ray when posture and metaball paramters change. This is much easier to model when using a combined formulation. A sketch of a definition of an alternative formulation that retains the modularity but allows for more complex modeling had been presented during the workshop. A description of this can be found in (Plänkers and Fua, 2001).

\section{Results}

The sequence in Figure 2(a) shows complex movements of a naked upper body, taken with a camera set up in front of the subject. Three cameras in an $L$ configuration were taking interlaced images at 20 frames/sec with an effective resolution of $432 \times 288$ per half-frame. Our stereo al- 

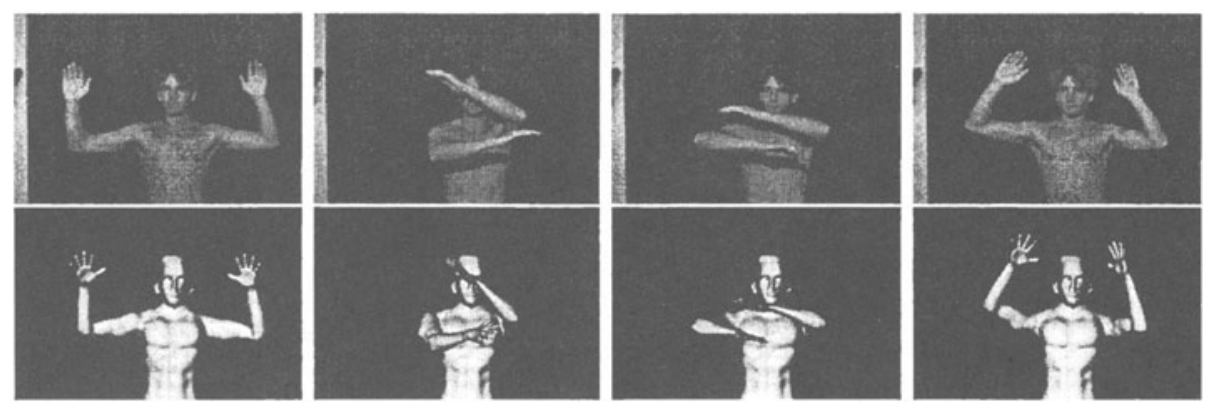

Figure 2. In the top row (a) is the original sequence of the upper body motion. Frames 10, 50, 60 and 90 out of 100 are shown. Results of the tracking and fitting with the animation model are shown in the bottom row with.

gorithm (Fua, 1995) produced very dense point clouds with about 4000 $3-\mathrm{D}$ points on the surface of the subject, even without textured clothes. To increase the frame rate and, thus, reduce the difference in posture between frames we used both halves of the interlaced images and adjusted the camera calibration accordingly.

The result of the tracking process is shown in Figure 2(b). The fitting step, using a more detailed model, produced slightly better postures, an adapted skeleton and resized metaballs (Fig. 2(c)). The head of this model was generated from a single video sequence of the subject by using the system of (Fua, 1999).

\section{Conclusion and Future Work}

We have presented a technique for fitting a complete animation model to image data and tracking complex 3-D motions. The model and the constraints it imposes are used to overcome the inherent noisiness of the data. We recover both motion and body shape from stereo video sequences. The corresponding parameters can be used to recreate realistic 3-D animations. Such a capability should be of great use in the area of human animation since it could also be used to analyze and visualize human motion for medical and training purposes. A more thorough description of our technique can be found in (Plänkers and Fua, 2001).

In future work, we intend to further exploit our strong model, for example the model can help to identify occlusions and decide whether to let the data guide the fitting or to let the prediction change the posture where no data is available. The model could also be used to derive an automatic and robust silhouette extraction algorithm, even with cluttered background. 
Baerlocher, P. and Boulic, R. (1999). Inverse kinematics report. Technical report, EPFL-DI-LIG.

Blanc, C. and Schlick, C. (1995). Extended field functions for soft objects. In Eurographics Workshop on Implicit Surfaces 95, pages 21-32, Grenoble, France.

Fua, P. (1995). Reconstructing Complex Surfaces from Multiple Stereo Views. In International Conference on Computer Vision, pages 1078-1085, Cambridge, MA.

Fua, P. (1999). Using Model-Driven Bundle-Adjustment to Model Heads from Raw Video Sequences. In International Conference on Computer Vision, Corfu, Greece.

Gavrila, D. (1999). The Visual Analysis of Human Movement: A Survey. Computer Vision and Image Understanding, 73(1).

Hilton, A., Beresford, D., Gentils, T., Smith, R., and Sun, W. (1999). Virtual People: Capturing Human Models to Populate Virtual Worlds. In Computer Animation, Geneva, Switzerland.

Konolige, K. (1997). Small Vision Systems: Hardware and Implementation. In Eighth International Symposium on Robotics Research, Hayama, Japan.

Lee, W., Gu, J., and Thalmann, N. M. (2000). Generating animatable 3d virtual humans from photographs. In Computer Graphics forum, Eurographics, volume 19, pages $\mathrm{C} 1-\mathrm{C} 10$, Interlaken, Switzerland.

Moeslund, T. and Granum, E. (2001). A Survey of Computer Vision-Based Human Motion Capture. Computer Vision and Image Understanding, 81(3).

Plänkers, R. and Fua, P. (2001). Articulated Soft Objects for Video-based Body Modeling. In International Conference on Computer Vision, Vancouver, Canada.

Press, W., Flannery, B., Teukolsky, S., and Vetterling, W. (1986). Numerical Recipes, the Art of Scientific Computing. Cambridge U. Press, Cambridge, MA.

Sullivan, S., Sandford, L., and Ponce, J. (1994). Using Geometric Distance Fits for 3-D. Object Modeling and Recognition. IEEE Transactions on Pattern Analysis and Machine Intelligence, 16(12):1183-1196.

Thalmann, D., Shen, J., and Chauvineau, E. (1996). Fast Realistic Human Body Deformations for Animation and VR Applications. In Computer Graphics International, Pohang, Korea. 